\title{
THE EXPERIMENTAL PRODUCTION OF ASCITES IN THE DOG WITH DIABETES INSIPIDUS ${ }^{1}$
}

\author{
By JOHN H. LARAGH, H. B. VAN DYKE, JULIUS JACOBSON, ${ }^{2}$ KARLIS ADAM- \\ SONS, JR., 3 AND STAMFORD L. ENGEL 4 \\ (From the Departments of Medicine, Pharmacology and Surgery, College of Physicians and \\ Surgeons, Columbia University and the Presbyterian Hospital in the City of New York.)
}

(Submitted for publication November 22, 1955; accepted April 3, 1956)

The role of the antidiuretic hormone of the neurohypophysis in the formation of edema remains to be clarified. Many investigators (1-4) have presented evidence which suggests that endogenous antidiuretic hormone may be responsible for the production and maintenance of various edematous states, especially the ascites of liver disease. The urine of patients with toxemia of pregnancy, nephritic edema, Cushing's syndrome, congestive heart failure, nephrosis, and cirrhosis of the liver with ascites, frequently has been found to contain increased amounts of an antidiuretic substance possibly secreted by the neurohypophysis.

Ralli and her group were perhaps the first of many workers to postulate a causal relationship between an increased liberation or persistence of antidiuretic hormone and the formation of ascites in hepatic cirrhosis $(5,6)$. As a result of studies on the control of ascites in this disease, Eisenmenger, Blondheim, Bongiovanni, and Kunkel (7, 8) attributed the beneficial effect of a dietary restriction of sodium to a decreased liberation of antidiuretic hormone. Shorr and his associates studied 23 patients with edema and found that the concentration of VDM (ferritin) in the blood was uniformly increased (9). These workers therefore postulated that the oliguria of heart failure, cirrhosis and nephrosis is the result of hypersecretion of antidiuretic hormone induced by VDM. In reviewing the problem of edema formation, Peters (10) pointed out that in the nephrotic syndrome as well as in cirrhosis and heart failure the

\footnotetext{
1 This work was supported in part by research grants from the U. S. Public Health Service, National Heart Institute (USPHS H-1275 and H-1788).

2 Research fellow of the Dazian Foundation.

3 Fellow of the National Foundation for Infantile Paralysis.

${ }^{4}$ Research Assistant, supported by a research grant (H-1788) from the U. S. Public Health Service, National Heart Institute.
}

serum sodium tends to be low. He believed that this hyponatremia is the result of a tendency to retain water in excess of salt and could be produced by an increased level of circulating antidiuretic hormone. Until recently, evidence that the antidiuretic hormone is inactivated by the normal liver had given implied support to the concept of abnormal persistence rather than hypersecretion of the hormone in cirrhosis (11). However, observation on patients (12) as well as in vitro studies (13) have failed to demonstrate inadequate hormone inactivation in this disease.

A major objection to the studies in which an increase in an antidiuretic factor in the urine was detected, is the failure to recognize the lack of specificity of the assay methods employed. van Dyke, Ames, and Plough (14) using a more specific intravenous assay in dogs to investigate the presence of antidiuretic hormone in the urine of patients with cirrhosis could not demonstrate a significant difference between a group with ascites and one without. In addition, in the various edematous states characterized by retention of salt and water, it is not clear whether the salt retention or the water retention occurs first.

The present study was designed to determine the role of the antidiuretic hormone in the formation of experimental ascites. In three dogs with known diabetes insipidus induced by interrupting the hypothalamico-hypophyseal tract in the hypothalamus, and in two control dogs, ascites was produced by constriction of the inferior vena cava above the hepatic vein by a modification of the method of McKee, Schilling, Tishkoff, and Hyatt (15). No significant difference was observed between normal dogs and dogs with diabetes insipidus in the capacity to form ascites after caval constriction. This report summarizes the results of electrolyte and water balance studies and of assays for anti- 


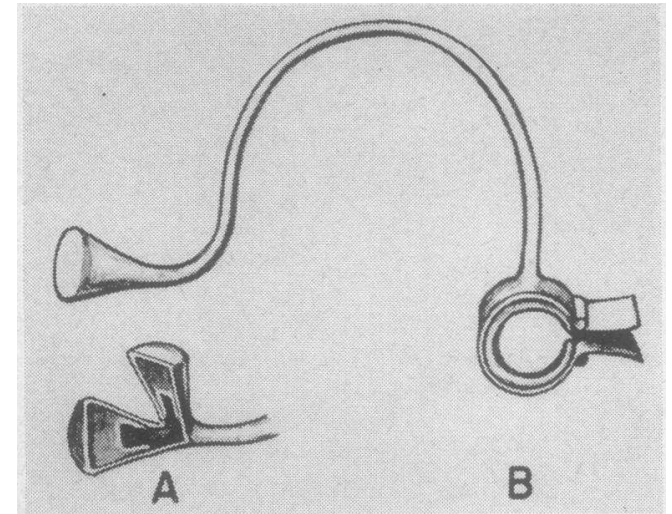

Fig. 1. Inflatable Cuff for Constriction of the Vena Cava. A. Section of Blind End Implanted Subcutaneously. B. Cuff Applied Around Vessel

diuretic hormone in the hypothalamus and posterior lobe of the pituitary.

\section{METHODS}

The experiments were carried out with trained unanesthetized mongrel dogs, housed in metabolism cages and fed a standard diet of fixed electrolyte content. Some dogs were fed "Kasco" dog food in known amounts, constant for a given dog. Others were fed ground beef with added sodium chloride. Each new batch of food was analyzed for its content of sodium, potassium, and chloride. The electrolyte content of any uneaten residue was determined and subtracted from the daily intake. Distilled water was given ad libitum, and the daily consumption was measured. Urine collections were made every twenty-four hours. Stool specimens were not analyzed. None of the dogs had diarrhea at any time. The dogs were weighed daily in the fasting state. Blood was taken from the jugular vein with a minimum of stasis and delivered under oil. The serum was analyzed for sodium, potassium, and chloride; total protein and urea nitrogen were determined by methods described previously $(16,17)$. The urine was analyzed daily for sodium, potassium, and chloride. The specific gravity of each twenty-four-hour sample was determined with a standard hydrometer.

Constriction of the vena cava was carried out after a control balance study of at least ten days, and the dogs were returned directly from the operating room to the metabolism cage. The constriction of the vena cava was accomplished by a modification of the method described by McKee, Schilling, Tishkoff, and Hyatt (15) using a pneumatic rubber cuff devised by one of the authors (J. J.) and shown in Figure 1. By injecting or withdrawing air from the rubber cuff through a blind end

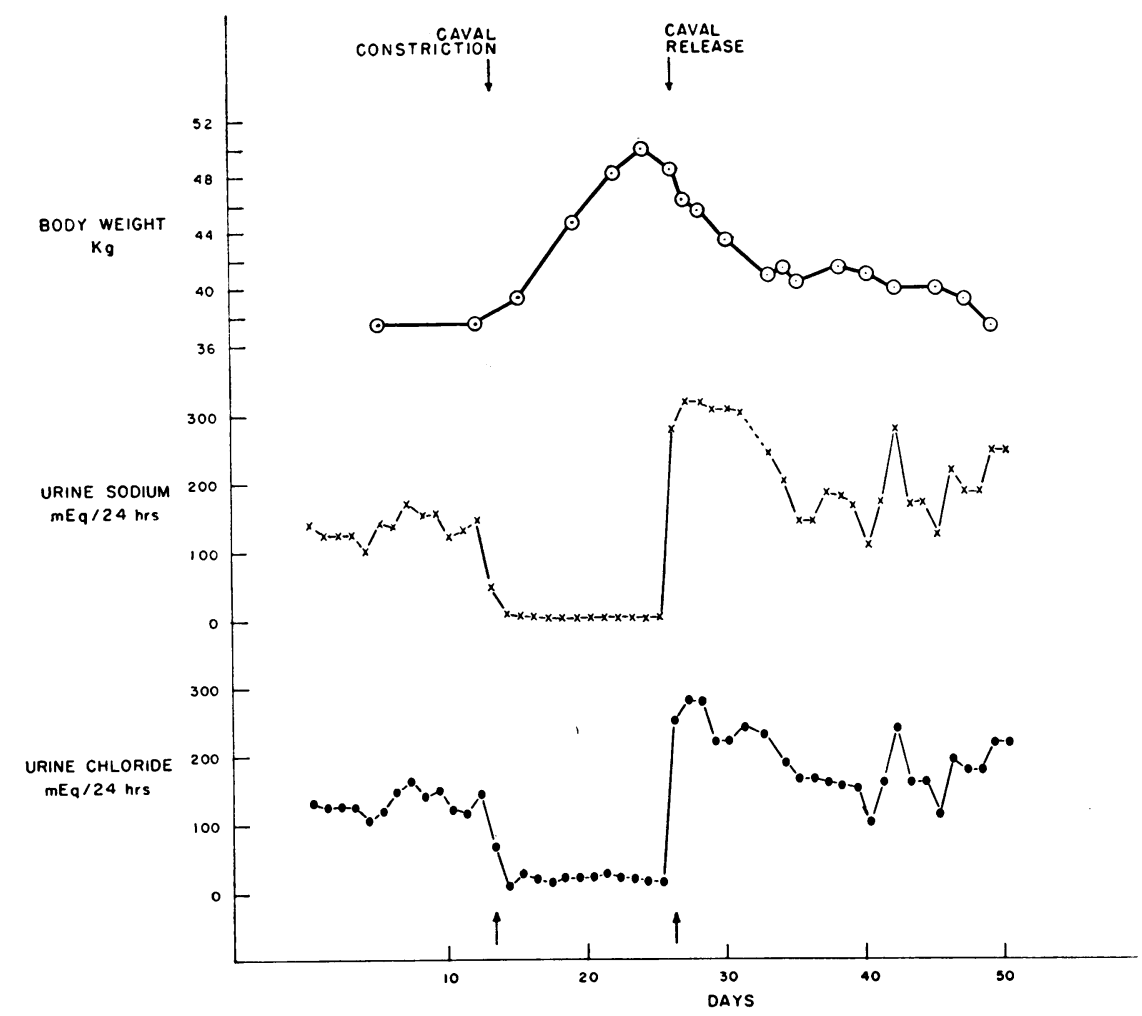

Fig. 2. Production of Reversible Edematous State in a Dog on Constant NaCl Intake 

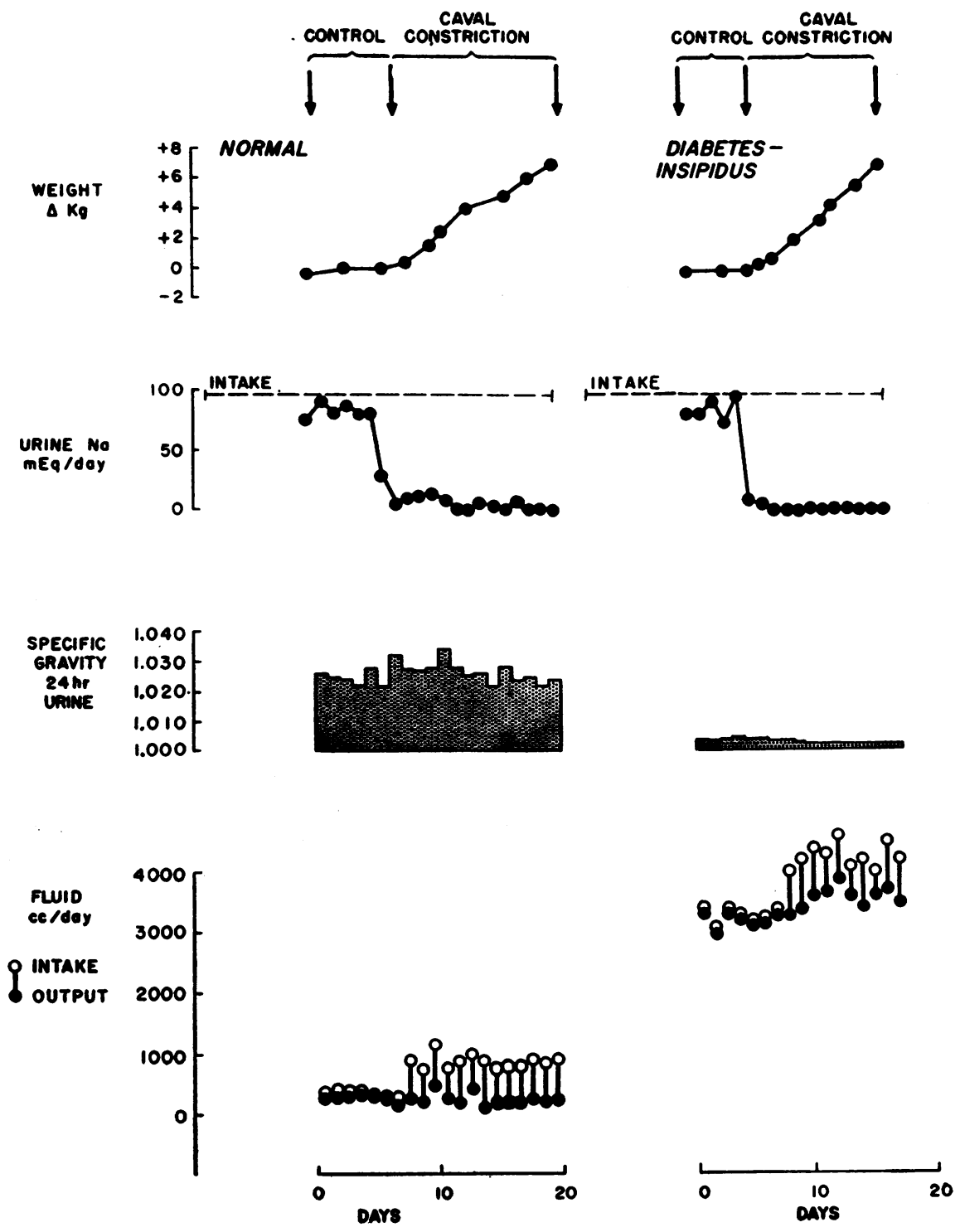

Fig. 3. Formation of Ascites in a Normal Dog and a Dog with Diabetes INSIPIDCS

implanted subcutaneously it is possible to produce a state of reversible, chronic ascites in the dog with diabetes insipidus (Figure 2) as well as in the control animals. Inflation and deflation of the previously inserted cuff through a No. 25 needle is accomplished without detectable evidence of disturbance of the animal. This cuff has been used in our laboratory to make controlled observations on the hormonal factors involved in the onset and offset of ascites characterized by renal retention of sodium chloride (18). After constriction of the vena cava, renal retention of sodium is almost immediately apparent and usually becomes maximal within the first twenty-four hours (Figure 2). After release of the constriction, an immediate natriuresis develops in which urine sodium may increase from less than $1 \mathrm{mEq}$. to over $300 \mathrm{mEq}$. in the first twenty-four hours. There is an associated diuresis and loss of edema fluid. Similar reversible edema occurs after pulmonary artery constriction, but this is technically more difficult to produce.

In three dogs diabetes insipidus had been induced by Dr. R. C. deBodo of the Department of Pharmacology, New York University College of Medicine, by means of a lesion produced by electrocoagulation in the anterior hypothalamus. Each dog excreted 3 to 6 liters of urine per twenty-four hours when having free access to food and water during the control periods. At the end of the study, the dogs were sacrificed, and the amount of antidiuretic hormone in the hypothalamus and in the pituitary was determined by a method similar to that of van Dyke, .Ames, and Plough (14). 


\section{RESULTS}

Following constriction of the thoracic inferior vena cava to less than half of its original diameter, ascites developed in the two normal control dogs. In both dogs the ascites was massive, requiring the removal of as much as 14 liters of fluid every four to six weeks. In one animal the ascites has persisted for over a year. Balance studies on both dogs (Figure 3 ) were similar to those previously reported in detail by others $(15,19)$.

Constriction of the thoracic vena cava in the three dogs with diabetes insipidus was also followed by the development of massive ascites. As indicated by the changes in body weight in two dogs, one diabetic and one with an intact neurohypophysis, presented graphically in Figure 3, ascitic fluid appeared to accumulate at the same rate in both groups following caval constriction. In both, maximal sodium retention developed after caval constriction. For example, the daily urine sodium content fell to a minimum of less than $1 \mathrm{mEq}$. per day in both studies presented in Figure 3 and never exceeded $8 \mathrm{mEq}$. per day. Striking differences were observed, however, in the urinary specific gravity and in the daily urine volume. In the dog with diabetes insipidus (Figure 3), urinary specific gravity did not rise above 1.006 , whereas in the normal control animal the specific gravity was always above 1.020 and reached a maximum of 1.037 . The dog with intact neurohypophysis (Figure 3 ) excreted small volumes ( 85 to $425 \mathrm{ml}$. per day) of urine despite a striking increase in thirst and water intake. In other normal dogs urine volumes as low as 25

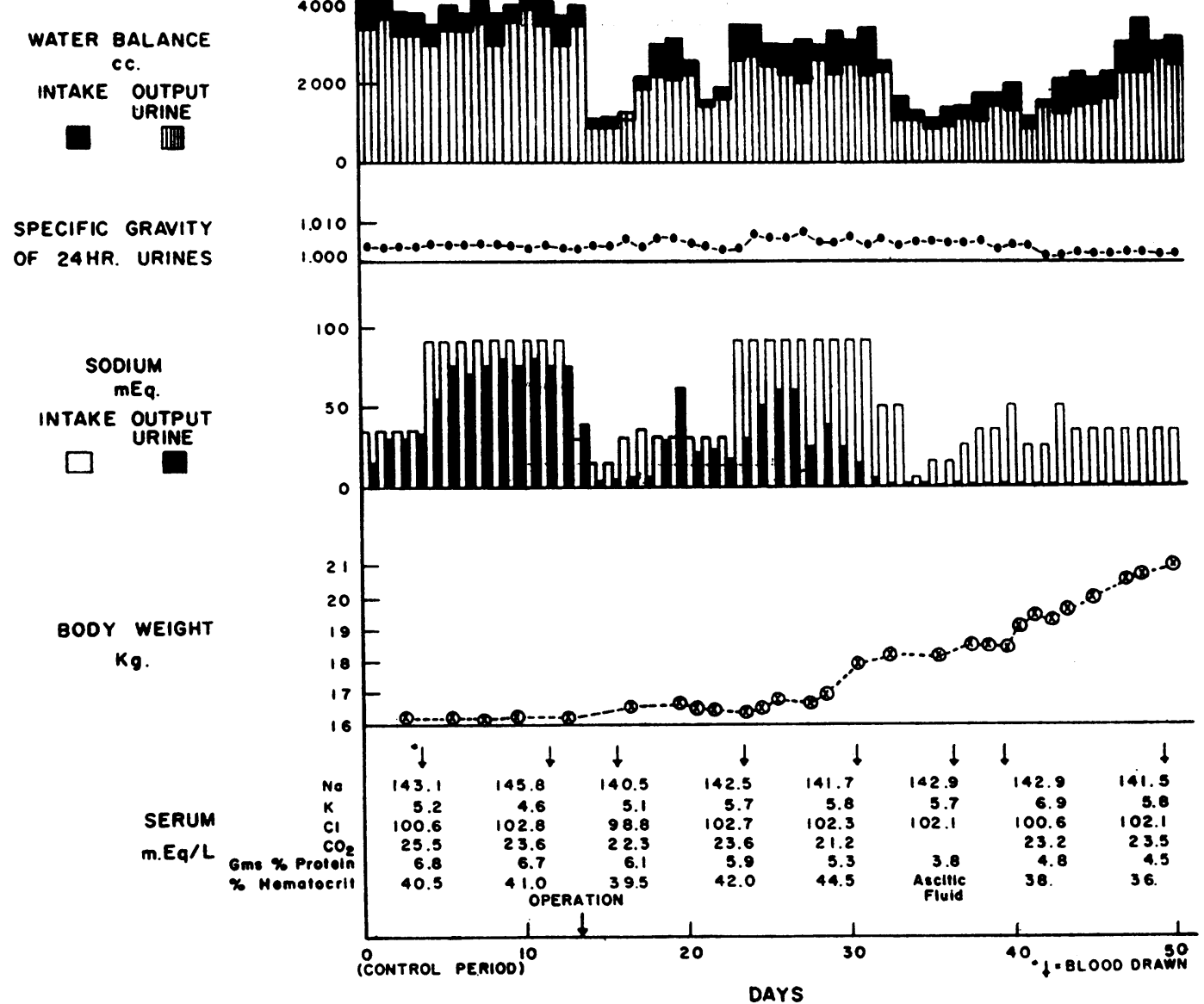

Fig. 4. The Production of Ascites in the Dog with Diabetes Insipidus 
ml. per twenty-four hours have at times been noted during ascites formation. In contrast, urinary output increased (3300 to $4000 \mathrm{ml}$. daily) in association with increased water drinking in the dog with diabetes insipidus during the formation of ascites (Figure 3). Despite the differences observed in water turnover and urine concentration the net water balance and resultant weight gain were comparable in the two dogs. In a second dog with diabetes insipidus, water turnover was reduced slightly during formation of ascites (Figure 4), and in the third animal it did not change appreciably. The control dog (Figure 3), on a constant diet containing $90 \mathrm{mEq}$. of sodium daily, gained $7.0 \mathrm{~kg}$. in the fifteen days following caval constriction. The diabetic dog received a diet containing $95 \mathrm{mEq}$. of sodium per day and gained $7.2 \mathrm{~kg}$. in the twelve days following constriction of the thoracic vena cava. These data, therefore, fail to show any significant difference in the rate of formation of ascitic fluid.

More detailed metabolic data for another diabetic dog are presented in Figure 4. This animal gained about $5 \mathrm{~kg}$. of ascites in a period of twentyfive days. Caval constriction was carried out during the operation instead of by subsequent inflation of the cuff. For some reason, urinary sodium retention did not immediately become maximal. The delay could be attributed to an inadequate constriction or perhaps to a post-operative reaction.

The urinary specific gravity in the dogs with diabetes insipidus ranged from 1.001 to 1.010 , remaining more or less fixed for a given animal. No appreciable changes in these values were observed during ascites formation. In the diabetic dog presented in Figure 3 a rapid accumulation of the ascitic fluid was evident while the specific gravity of the urine remained constant at 1.004 . During this phase of maximal production of ascitic fluid, almost all of the administered sodium was retained. A corresponding reduction in chloride excretion was consistently observed in all animals.

The serum sodium concentration of the diabetic dogs developing ascites remained relatively constant in the normal range. In one of the normal dogs, the serum sodium also remained constant, but in the other control dog forming ascites the serum sodium fell to $138 \mathrm{mEq}$. per L. from 145 mEq. per L. This tendency to hyponatremia in
TABLE I

The total amount of antidiuretic hormone in the neurohypophysis of the dogs with diabetes insipidus in comparison with normal dogs *

\begin{tabular}{cccc}
\hline & \multicolumn{3}{c}{ Total mU of ADH } \\
\cline { 2 - 4 } Dog No. & $\begin{array}{c}\text { Hypo- } \\
\text { thalamus }\end{array}$ & $\begin{array}{c}\text { Posterior } \\
\text { lobe }\end{array}$ & $\begin{array}{c}\text { Total in } \\
\text { neuro- } \\
\text { hypophysis }\end{array}$ \\
\hline 1 & 67 & 60 & 127 \\
2 & 0 & 19 & 19 \\
3 & 160 & 725 & 885 \\
Normal & 4,060 & 16,700 & 20,760 \\
(Av. of & & & \\
11 dogs) $\dagger$ & & &
\end{tabular}

* Corresponding metabolic data for dogs 1, 2, 3 are summarized in Figures $2,3,4$, respectively.

$\dagger$ van Dyke, Adamsons, and Engel (4).

ascitic dogs has also been observed at times by Davis and Howell (19). Schilling and associates (20) and Davis and Howell (19) have also noted a definite tendency to hyperkalemia in normal dogs with ascites following caval constriction. We have observed a similar change in diabetic dogs with ascites, an example of which is presented in Figure 4. Serum potassium values in excess of $7.0 \mathrm{mEq}$. per L. were encountered in our animals on five occasions. Periods of rapid edema formation were associated with a striking fall in the serum protein concentration in both the normal and the diabetic dogs. The protein content of the ascitic fluid was found to range from 1.6 to 4.4 gm. per $100 \mathrm{ml}$. in both groups of animals. Tests of liver function were made in two dogs with ascites, one control and one dog with diabetes insipidus. The protein partition, alkaline phosphatase and serum bilirubin were all found to be well within normal limits, despite enlargement of the liver and gross evidences of cirrhosis after prolonged caval constriction.

At the time of sacrifice or death, the hypothalamus and the atrophic posterior pituitary of the three dogs with diabetes insipidus were assayed for antidiuretic hormone (Table I). The assays were performed by intravenous injection of tissue extracts into heavily hydrated dogs. Less than $0.5 \mathrm{mU}$ can be detected by this method. Owing to post-operative scarring of the hypothalamus, it was not feasible to assay separately some of the divisions of the neurohypophysis such as the hypothalamico-hypophyseal tract in the tuber cinereum or the paired supraoptic and paraven- 
tricular nuclei. The pituitary body was assayed without separation of the lobes. The results in comparison with a group of eleven normal dogs are summarized in Table I. Except in dog No. 3 (Figure 4), only about 4 per cent of the total amount normally present could be detected in the neurohypophysis (hypothalamus and posterior pituitary). Thus, only trivial amounts of stored antidiuretic hormone could be detected in the neurohypophysis of dogs 1 and 2 . The reduction in stored hormone was severe but less pronounced in dog 3. The quantities of stored hormone present do not furnish information concerning the rate of formation or liberation of antidiuretic hormone. However, the characteristic polyuria of these animals in association with low or very low neurohypophyseal titers of stored hormone suggests that the formation and liberation of $\mathrm{ADH}$ were grossly inadequate.

\section{DISCUSSION}

Several groups of investigators have previously characterized the metabolic events accompanying the onset and termination of ascites formation after constriction of the thoracic vena cava $(15,19$ 21). Such animals exhibit chronic fluid retention with an expanded extracellular fluid volume as a result of more complete reabsorption of sodium by the renal tubules. At least in this important respect, dogs with experimental ascites are similar to patients with cardiac failure and decompensated hepatic cirrhosis. Further studies of Davis, Howell, Southworth, and Laqueur (22, 23) have shown that this type of edema usually clears after adrenalectomy or anterior hypophysectomy. Their data indicate that a salt-retaining hormone of the adrenal is essential for the edema formation and that anterior pituitary activity appears to contribute to ascitic fluid accumulation by maintaining an adequate level of venous hypertension.

The present study clearly demonstrates that ascitic fluid is formed readily in the dog following constriction of the thoracic inferior vena cava with storage of little or very little antidiuretic hormone in the neurohypophysis. Since the very low amounts of stored hormone could be correlated with the excretion of abnormally great volumes of urine, it is presumed that very little antidiu- retic hormone could be liberated by the dogs with diabetes insipidus. The ascites of diabetic dogs appears to be similar to the ascites produced in normal dogs, and the rate of its formation was not shown to be appreciably different. In both groups of animals the formation of ascitic fluid is characterized by maximal renal retention of ingested sodium; water and chloride are apparently retained in amounts sufficient to sustain osmolarity of body fluid.

The formation of ascites in dogs with diabetes insipidus appears to differ from that observed in control animals only in that it is characterized by persistence of a dilute urine of large volume. The presence of an intact neurohypophysis would thus appear to account for the comparatively low water turnover noted in the normal controls. It is implied in this, although not established, that overactivity of the antidiuretic hormone could account for oliguria, retention of water in excess of salt and resultant hyponatremia seen at times in the control dogs and in certain patients with ascites.

Animals with diabetes insipidus have an inherently greater tendency to a negative water balance. In this study ascites formation was related to the amount of sodium ingested but was largely independent of water ingested. It is therefore suggested that abnormal retention of sodium and chloride rather than of water is of prime importance in the accumulation of ascitic fluid after constriction of the vena cava.

\section{SUMMARY}

Progressive ascites was readily produced by constriction of the inferior vena cava at the level of the diaphragm in two normal control dogs and in three dogs with diabetes insipidus. The diabetes insipidus had been induced previously by bilateral destruction of the neurohypophyseal tracts.

The rate of formation of ascitic fluid after caval constriction was similar in both groups.

At subsequent postmortem little or no antidiuretic hormone could be demonstrated in the neurohypophysis of the diabetic animals.

During ascites formation in normal dogs urine formation tended to decrease, and the urine tended to become more concentrated despite an increase in water intake. On the other hand, among the 
diabetic dogs, polydypsia with the continued excretion of large volumes of dilute urine persisted during accumulation of ascitic fluid.

In both groups of animals, the formation of ascites was characterized by nearly maximal renal retention of ingested sodium.

\section{REFERENCES}

1. Smith, H. W., The Kidney: Structure and Function in Health and Disease. New York, Oxford University Press, 1951, pp. 264-267.

2. van Dyke, H. B., Antidiuretic factors. Transactions, 2nd Josiah Macy Jr. Conf. on Renal Function, 1950, p. 48.

3. Pickford, M., Antidiuretic substances. Pharmacol. Rev., 1952, 4, 254.

4. van Dyke, H. B., Adamsons, K., Jr., and Engel, S. L., Aspects of the biochemistry and physiology of the neurophyseal hormones. Recent Progress in Hormone Research, 1955, 11, 1.

5. Ralli, E. P., Robson, J. S., Clarke, D., and Hoagland, C. L., Factors influencing ascites in patients with cirrhosis of the liver. J. Clin. Invest., 1945, 24, 316.

6. Ralli, E. P., Leslie, S. H., Stueck, G. H., Jr., and Laken, B., Studies of the serum and urine constituents in patients with cirrhosis of the liver during water tolerance tests. Am. J. Med., 1951, 11, 157.

7. Eisenmenger, W. J., Blondheim, S. H., Bongiovanni, A. M., and Kunkel, H. G., Electrolyte studies on patients with cirrhosis of the liver. J. Clin. Invest., 1950, 29, 1491.

8. Eisenmenger, W. J., Role of sodium in the formation and control of ascites in patients with cirrhosis. Ann. Int. Med., 1952, 37, 261.

9. Shorr, E., Baez, S., Zweifach, B. W., Payne, M. A., and Mazur, A., The antidiuretic action of the hepatic vasodepressor ferritin (VDM) and its occurrence in conditions associated with antidiuresis in man. Tr. A. Am. Physicians, 1950, 63, 39.

10. Peters, J. P., The problem of cardiac edema. Am. J. Med., 1952, 12, 66.

11. Birnie, J. H., The inactivation of posterior pituitary antidiuretic hormone by liver extracts. Endocrinology, 1953, 52, 33.
12. Bernstein, S. H., Weston, R. E., Ross, G., Grossman, J., Hanenson, I. B., and Leiter, L., Studies on intravenous water diuresis and nicotine and pitressin antidiuresis in normal subjects and patients with liver disease. J. Clin. Invest., 1953, 32, 422.

13. Miller, G. E., and Townsend, C. E., The hepatic inactivation of antidiuretic hormone. J. Clin. Invest., 1953, 32, 590.

14. van Dyke, H. B., Ames, R. G., and Plough, I. C., The excretion of antidiuretic hormone in the urine of patients with cirrhosis of the liver. $\mathrm{Tr}$. A. Am. Physicians, 1950, 63, 35.

15. McKee, F. W., Schilling, J. A., Tishkoff, G. H., and Hyatt, R. E., Experimental ascites: Effects of sodium chloride and protein intake on protein metabolism of dogs with constricted inferior vena cava. Surg., Gynec. \& Obst., 1949, 89, 529.

16. Laragh, J. H., The effect of potassium chloride on hyponatremia. J. Clin. Invest., 1954, 33, 807.

17. Laragh, J. H., and Capeci, N. E., Effect of administration of potassium chloride on serum sodium and potassium concentration. Am. J. Physiol., 1955, 180, 539.

18. Jacobson, J., and Laragh, J. H., To be published.

19. Davis, J. O., and Howell, D. S., Mechanisms of fluid and electrolyte retention in experimental preparations in dogs. II. With thoracic inferior vena cava constriction. Circ. Research, 1953, 1, 171.

20. Schilling, J. A., McCoord, A. B., Clausen, S. W., Troup, S. B., and McKee, F. W., Experimental ascites. Studies of electrolyte balance in dogs with partial and complete occlusion of the portal vein and of the vena cava above and below the liver. J. Clin. Invest., 1952, 31, 702.

21. Hyatt, R. E., and Smith, J. R., The mechanism of ascites. A physiologic appraisal. Am. J. Med., 1954, 16, 434.

22. Davis, J. O., Howell, D. S., and Southworth, J. L., Mechanisms of fluid and electrolyte retention in experimental preparations in dogs, III. Effect of adrenalectomy and subsequent desoxycorticosterone acetate administration on ascites formation. Circ. Research, 1953, 1, 260.

23. Howell, D. S., Davis, J. O., and Laqueur, G. L., Effect of hypophysectomy on electrolyte excretion in dogs with ascites produced by thoracic inferior vena cava constriction. Circ. Research, 1955, 3, 264. 